\title{
Post-traumatic stress disorder in the context of terrorism and other civil conflict in Northern Ireland: randomised controlled trial
}

\author{
Michael Duffy, ${ }^{1}$ Kate Gillespie,, ${ }^{2}$ David M Clark ${ }^{3}$
}

\section{EDITORIAL by McDonald}

'University of Ulster at Magee, Londonderry, Northern Ireland BT48 7)L

${ }^{2}$ Northern Ireland Centre for Trauma and Transformation, Omagh, Northern Ireland

${ }^{3}$ King's College London

Correspondence to: M Duffy m.duffy1@ulster.ac.uk

BMJ 2007;334:1147-50 doi: 10.1136/bmj.39021.846852.BE
This article is an abridged version of a paper that was published on bmj.com on 11 May 2007. Cite this version as: $B M J$ 11 May 2007, doi: $10.1136 /$ bmj.39021.846852.BE (abridged text, in print: BMJ 2007;334:1147-50)

\begin{abstract}
Objective To evaluate the effectiveness of cognitive therapy for post-traumatic stress disorder related to terrorism and other civil conflict in Northern Ireland. Design Randomised controlled trial.
\end{abstract}

Setting Community treatment centre, Northern Ireland. Participants 58 consecutive patients with chronic posttraumatic stress disorder (median 5.2 years, range 3 months to 32 years) mostly resulting from multiple traumas linked to terrorism and other civil conflict. Interventions Immediate cognitive therapy compared with a waiting list control condition for 12 weeks followed by treatment. Treatment comprised a mean of 5.9 sessions during 12 weeks and 2.0 sessions thereafter. Main outcome measures Primary outcome measures were patients' scores for post-traumatic stress disorder (post-traumatic stress diagnostic scale) and depression (Beck depression inventory). The secondary outcome measure was scores for occupational and social functioning (work related disability, social disability, and home life) on the Sheehan disability scale.

Results At 12 weeks after randomisation, immediate cognitive therapy was associated with significantly greater improvement than the waiting list control group in the symptoms of post-traumatic stress disorder (mean difference $9.6,95 \%$ confidence interval 3.6 to 15.6 ), depression (mean difference 10.1, 4.8 to 15.3), and self reported occupational and social functioning (mean difference 1.3, 0.3 to 2.5). Effect sizes from before to after treatment were large: post-traumatic stress disorder 1.25, depression 1.05, and occupational and social functioning 1.17. No change was observed in the control group.

Conclusion Cognitive therapy is an effective treatment for post-traumatic stress disorder related to terrorism and other civil conflict.

Trial registration Current Controlled Trials ISRCTN16228473.

\section{INTRODUCTION}

Little is known about how to successfully treat trauma resulting from terrorist related events. The only published evaluation of treatment after a terrorist bomb is a trial of cognitive therapy for post-traumatic stress disorder after the car bomb in Omagh, Northern Ireland, in 1998. In this uncontrolled study, ${ }^{1}$ cognitive therapy delivered up to two years after the event was associated with improvements in post-traumatic stress disorder as large as those observed with cognitive therapy in randomised controlled trials of non-terrorism related posttraumatic stress disorder. The Northern Ireland Centre for Trauma and Transformation was therefore set up to offer trauma focused cognitive therapy to people in Northern Ireland affected by terrorism and other civil conflict over the past four decades. We evaluated the effectiveness of that therapy.

\section{METHODS}

We invited patients referred to the Northern Ireland Centre for Trauma and Transformation between August 2003 and September 2004 and meeting diagnostic criteria for post-traumatic stress disorder after traumatic events in adulthood to participate in the trial. They were randomly allocated to immediate cognitive therapy or to a 12 week wait followed by cognitive therapy. Treatment involved up to one therapy session a week for 12 weeks, followed by additional sessions if needed. Primary outcome measures were severity of post-traumatic stress disorder (posttraumatic stress diagnostic scale) and severity of depression (Beck depression inventory). ${ }^{23}$ The secondary outcome measure was the Sheehan disability scale,${ }^{4}$ with separate items for work, social, and family related disability. We obtained scores for each measure before treatment or wait, after 12 weeks (end of wait), after treatment, and at 1, 4, and 12 months' follow-up. Patients also completed the post-traumatic stress diagnostic scale and Beck depression inventory before each session.

To determine whether cognitive therapy is effective, at 12 weeks we compared the immediate cognitive therapy and waiting list groups' scores for posttraumatic stress disorder, depression, and functioning (work related disability, social disability, and family related disability). To determine the overall improvement that could be obtained with cognitive therapy, we compared scores before treatment and after treatment, combining the immediate treatment and delayed treatment groups.

To estimate the magnitude of the changes in scores from before to after treatment we calculated effect 
sizes: small (0.20-0.49), medium (0.50-0.79), and large $(\geq 0.8$, see bmj.com). We also calculated residualised gain scores, which control for variation in baseline levels, and used them to explore possible predictors of improvement, including therapist effects.

The cognitive therapy programme is described elsewhere. ${ }^{15}$ Five therapists delivered the treatment. We used behavioural activation early in therapy to lift mood when high initial levels of depression interfered with trauma processing.

\section{Statistical analysis}

We carried out analyses on an intention to treat basis and on patients who completed the study, including patients in the waiting list group who subsequently completed cognitive therapy. Patients were classified as having completed the study if they had at least six therapy sessions or they ended therapy earlier as agreed by the therapist. Comparisons between the immediate treatment and waiting list groups at 12 weeks were based on analyses of covariance, with scores before treatment or wait as covariates. We used $t$ tests to compare scores before treatment, after treatment, and at follow-up. Number of traumas and years since the traumatic event were not normally distributed and were transformed before analysis. Preliminary analyses included drug status at intake and several patient characteristics (civilian, experienced bombing, taken hostage) as factors. None of these was related to outcome. We calculated percentage change in scores for post-traumatic stress disorder from before to after treatment for all treated patients.

\section{RESULTS}

Fifty eight patients met the criteria for post-traumatic stress disorder (see bmj.com): 29 were allocated to immediate cognitive therapy and 29 to a 12 week wait then cognitive therapy (control group). The groups were similar at baseline (see bmj.com). Most patients had experienced multiple traumatic events.

The mean (standard deviation) number of treatment sessions was $7.8(5.1)$, with $5.9(2.5)$ sessions in the first 12 weeks and 2.0 (3.2) sessions thereafter. Twelve patients $(21 \%)$ dropped out. The mean (standard deviation) numbers of sessions for those who dropped out and those who completed the study were 2.8 (1.2) and $9.2(4.9)$.
At 12 weeks the scores for post-traumatic stress disorder, depression, and functioning were significantly lower in the immediate therapy group (see bmj.com). The wait group did not improve on any measure whereas the immediate therapy group improved on all measures $(\mathrm{P}<0.001$, paired $t$ tests $)$.

The table shows the scores for patients who received cognitive therapy. The groups were combined as the control group showed no improvement during wait and did not differ in improvement during treatment from the immediate therapy group. Twenty seven of 57 patients $(47 \%)$ had additional sessions. The overall improvements in scores for post-traumatic stress disorder, depression, and functioning were greater than those at 12 weeks. Paired $t$ tests showed highly significant reductions between the scores before and after treatment. The observed effect sizes for post-traumatic stress disorder, depression, and functioning (work, social, and family) for patients who completed the study were $1.74,1.24,1.08,1.36$, and 0.96 (intention to treat sample $1.25,1.05,0.97,1.03$, and 0.70 ).

Overall 43 patients $(96 \%)$ who completed treatment provided follow-up data (table). Treatment gains were well maintained at follow-up. No significant difference was found in the scores at follow-up for post-traumatic stress disorder, depression, and social functioning. Further significant improvement was evident at follow-up for work related disability $(\mathrm{P}<0.05)$ and family related disability $(\mathrm{P}<0.01)$. Effect sizes from before treatment to follow-up were large for all measures (see bmj.com).

Percentage change in post-traumatic stress disorder scores from before to after treatment showed that four (out of 57) patients deteriorated and four showed no change. Deterioration occurred more often during the wait period than during therapy $(38 \% v 7 \%)$.

Psychiatric comorbidity and enduring physical health problems as a consequence of the trauma were not related to degree of improvement. However, higher scores for depression at baseline $(\mathrm{P}<0.05)$ and a longer time since the trauma $(\mathrm{P}<0.05)$ were associated with less improvement.

A significant therapist effect was found when residualised gain scores were compared between therapists. Paired comparisons indicated significant differences $(\mathrm{P}<0.05)$ between each of the two therapists whose patients had improved most and one therapist whose patients had improved least. The patients

Mean (standard deviation) scores before treatment, after treatment, and at follow-up (immediate and delayed cognitive therapy groups combined)

\begin{tabular}{|c|c|c|c|c|c|}
\hline \multirow[b]{2}{*}{ Measure } & \multicolumn{3}{|c|}{ Completers $(n=45)^{\star}$} & \multicolumn{2}{|c|}{ All patients $(n=57) \dagger$} \\
\hline & Before treatment & After treatment & Follow-up & Before treatment & After treatment \\
\hline Post-traumatic diagnosis scale & $33.9(10.1)$ & $14.6(12.0)$ & $12.6(11.1)$ & $34.3(9.6)$ & $18.9(14.5)$ \\
\hline Beck depression inventory & $33.3(12.4)$ & $17.5(13.0)$ & $14.9(12.9)$ & $34.6(12.1)$ & $20.8(14.2)$ \\
\hline Work related disability & $7.8(2.6)$ & $4.6(3.3)$ & $3.6(3.2)$ & $8.1(2.4)$ & $5.3(3.3)$ \\
\hline Social life related disability & $7.7(2.5)$ & $4.1(2.8)$ & $3.6(3.1)$ & $7.7(2.4)$ & $4.9(3.0)$ \\
\hline Family related disability & $6.8(2.7)$ & $4.1(2.9)$ & $3.1(3.0)$ & $6.8(2.7)$ & $4.8(3.0)$ \\
\hline
\end{tabular}

*43 patients completed at least one follow-up assessment. Post-treatment scores were carried forward for remaining two patients.

tTreatment drop-outs were not followed up. One patient showed substantial improvement during wait and declined subsequent treatment. 
treated by these two sets of therapists did not differ in symptoms of initial post-traumatic stress disorder, initial depression, or time since the trauma, but patients treated by the therapist with poorer outcomes were significantly older $(\mathrm{P}<0.01)$ and had significantly fewer treatment sessions $(\mathrm{P}<0.01)$. Multiple regression indicated that the therapist effect remained significant after controlling for these variables.

The drop-out rate $(20 \%)$ was higher than that in the two previous randomised controlled trials ${ }^{67}$ of cognitive therapy for post-traumatic stress disorder $(0 \%-5 \%)$, both of which focused on non-terrorism related trauma.

\section{DISCUSSION}

Cognitive therapy is an effective treatment for posttraumatic stress disorder related to terrorism and other civil conflict. Patients allocated to immediate therapy showed significant and substantial reductions in the symptoms of post-traumatic stress disorder and depression and noticeable improvements in self reported functioning (work, social, and family related). Patients allocated to wait (control group) showed no change.

The trial extends the findings of the Omagh bomb study. ${ }^{1}$ Both studies had minimal exclusion criteria and so are best conceptualised as effectiveness studies. However, patients in the Omagh bomb study were treated between three months and two years after the bombing, whereas patients in the current study were treated up to 33 years after trauma (median 6 years). Half had failed other treatments and many had high levels of depression.

Unlike the two randomised controlled trials ${ }^{67}$ of $\operatorname{cog}$ nitive therapy for non-terrorism related post-traumatic stress disorder, which were restricted to patients who had experienced no more than two major traumas, $59 \%$ of patients in the present study had experienced more than two traumas. Such patients improved as much as those who had experienced fewer traumas.

Although cognitive therapy was effective overall, the degree of improvement varied, which suggested several important things. Firstly, psychiatric comorbidity did not influence the extent of reduction in symptoms of post-traumatic stress disorder. This may be because study therapists were given flexibility in the number of sessions that they could offer and were also allowed to utilise cognitive behaviour therapy techniques for other conditions. Patients with comorbidities received more sessions than those without comorbidities.

Secondly, in contrast to the Omagh bomb study, ${ }^{1}$ ongoing physical problems resulting from the trauma did not predict poorer outcome. This may be because patients had longer to adapt to the problem. Also, the team may have become better at dealing with problems as a result of treating victims of the Omagh bombing.

Thirdly, high levels of depression at intake were associated with poorer outcome. This was not observed in the Omagh bomb study ${ }^{1}$ or in the two

\section{WHAT IS ALREADY KNOWN ON THIS TOPIC}

Trauma focused cognitive behaviour therapy and eye movement desensitisation and reprocessing therapy are recommended by NICE for non-terrorism related posttraumatic stress disorder

\section{WHAT THIS STUDY ADDS}

Cognitive therapy is effective in the treatment of posttraumatic stress disorder related to terrorism and other civil conflict

randomised controlled trials. ${ }^{67}$ However, the average scores for depression at intake were higher in the present study (average 35) than in the other studies (28, 19, and 24). Furthermore, mean scores at intake were as high as for published trials of severe major depression. ${ }^{8}$ We included behavioural activation to help reduce high levels of initial depression.

Fourthly, the drop-out rate $(20 \%)$ was higher than those reported in the previous three studies of this cognitive therapy programme but is not unusually high for trauma focused cognitive behaviour therapy programmes in general (see bmj.com). The variation in outcome associated with different therapists (14\%) was much larger than that observed in a randomised controlled trial of the same treatment $(<1 \%)$, run in a university research clinic with therapists who had received extensive training to protocol.

In view of recent NICE guidelines, NHS trusts are likely to make cognitive behaviour therapy available to a variety of populations. When the Northern Ireland Centre for Trauma and Transformation was established, evidence was not available to indicate whether the positive results that have been observed with cognitive behaviour for non-terrorism post-traumatic stress disorder would generalise to chronic terrorist and civil conflict related violence. The centre established its treatment programme as a randomised controlled trial, with referred adults with post-traumatic stress disorder. In this way it was possible to determine in a relatively short time that treatment is generalisable.

\section{Limitations}

Our study relied on self report measures of posttraumatic stress disorder and associated symptoms. We do not think that this compromised the study because in two previous trials ${ }^{67}$ of cognitive therapy for post-traumatic stress disorder and in several trials of cognitive behaviour therapy for other anxiety disorders, ${ }^{910}$ assessor ratings and self report scores were similar. If there was any demand effect for self reported symptoms it might be more in the direction of under-reporting of improvement at the end of the controlled phase of the study (12 weeks). This is because patients who had reported only modest improvement were offered further treatment.

The therapists were Sean Collins, MD, Kate Gillespie, Susan McGandy, and John McLaren. Tracy McCrossan assisted with data collection. DMC is supported by 
the Wellcome Trust. We thank David Bolton, director of the Northern Ireland Centre for Trauma and Transformation, for supporting the research.

Contributors: See bmj.com.

Funding: Northern Ireland Victims Liaison Unit.

Competing interests: None declared.

Ethical approval: This study was approved by the ethics committee of

Queen's University of Belfast School of Medicine.

1 Gillespie K, Duffy M, Hackmann A, Clark DM. Community based cognitive therapy in the treatment of post-traumatic stress disorder following the Omagh bomb. Behav Res Ther 2002;40:345-57.

2 Foa EB, Cashman L, Jaycox L, Perry K. The validation of a self-report measure of posttraumatic stress disorder: the posttraumatic diagnostic scale. Psychol Assess 1997;9:445-51.

3 Beck AT, Rush AJ, Shaw BF, Emery G. Cognitive therapy of depression. New York: Guilford Press, 1979.

4 Sheehan DV. The anxiety disease. New York: Scribner, 1983.

5 Clark DM, Ehlers A. Posttraumatic stress disorder: from theory to therapy. In: Leahy RL, ed. Contemporary cognitive therapy. New York: Guilford, 2004:141-60.
6 Ehlers A, Clark DM, Hackmann A, McManus F, Fennell M, Herbert C, et al. A randomized controlled trial of cognitive therapy, a self-help booklet, and repeated assessments as early interventions for posttraumatic stress disorder. Arch Gen Psychiatry 2003;60:1024-32

7 Ehlers A, Clark DM, Hackmann A, McManus F, Fennell M. Cognitive therapy for post-traumatic stress disorder: development and evaluation. Behav Res Ther 2005;43:413-31.

8 DeRubeis RJ, Gelfand LA, Tang TZ, Simons AD. Medications versus cognitive behavior therapy for severely depressed outpatients: mega-analysis of four randomized comparisons. Am J Psychiatry 1999;156:1007-13.

9 Clark DM, Salkovskis PM, Hackmann A, Middleton $\mathrm{H}$, Anastasiades P, Gelder MG. A comparison of cognitive therapy, applied relaxation and imipramine in the treatment of panic disorde. Brit J Psychiatry 1994;164:759-69.

10 Clark DM, Ehlers A, Hackmann A, McManus F, Fennell MJV, Waddington L, et al. Cognitive therapy versus exposure plus applied relaxation in social phobia: a randomised controlled trial. J Consult Clin Psychol 2006;74:568-78.

Accepted: 22 March 2007

\title{
Effect of nationwide injury prevention programme on serious spinal injuries in New Zealand rugby union: ecological study
}

\author{
Kenneth L Quarrie, ${ }^{1}$ Simon M Gianotti, ${ }^{2}$ Will G Hopkins, ${ }^{3}$ Patria A Hume ${ }^{4}$
}

\section{EDITORIAL by Noakes and Draper}

${ }^{1}$ Research \& Injury Prevention, New Zealand Rugby Union, PO Box 2172, Wellington, New Zealand

${ }^{2}$ Sport \& Road, Accident

Compensation Corporation, Wellington

${ }^{3}$ Exercise Science, Institute of Sport \& Recreation Research New Zealand, Faculty of Health and

Environmental Science,

AUT University, Auckland 1020 New Zealand

${ }^{4}$ Institute of Sport \& Recreation Research New Zealand

Correspondence to: K Quarrie

ken.quarrie@nzrugby.co.nz

BMJ 2007:334:1150-3

doi: 10.1136/bmj.39185.605914.AE

This article is an abridged version of a paper that was published on bmj.com on 18 May 2007.

Cite this version as: $B M$

18 May 2007, doi: $10.1136 /$

bmj.39185.605914.AE

(abridged text, in print: $B M$ J

2007;334:1150-3)

\section{ABSTRACT}

Objective To investigate the effect of RugbySmart, a nationwide educational injury prevention programme, on the frequency of spinal cord injuries.

Design Ecological study.

Setting New Zealand rugby union.

Participants Population at risk of injury comprised all New Zealand rugby union players.

Intervention From 2001, all New Zealand rugby coaches and referees have been required to complete RugbySmart, which focuses on educating rugby participants about physical conditioning, injury management, and safe techniques in the contact phases of rugby.

Main outcome measures Numbers of all spinal injuries due to participation in rugby union resulting in permanent disablement in 1976-2005, grouped into five year periods; observed compared with predicted number of spinal injuries in 2001-5.

Results Eight spinal injuries occurred in 2001-5, whereas the predicted number was 18.9 (relative rate $=0.46,95 \%$ confidence interval 0.19 to 1.14$)$. Only one spinal injury resulted from scrums over the period; the predicted number was 9.0 (relative rate $=0.11,0.02$ to 0.74 ). Corresponding observed and predicted rates for spinal injuries resulting from other phases of play (tackle, ruck, and maul) were 7 and 9.0 (relative rate $=0.83,0.29$ to 2.36).

Conclusions The introduction of the RugbySmart programme coincided with a reduction in the rate of disabling spinal injuries arising from scrums in rugby union. This study exemplifies the benefit of educational initiatives in injury prevention and the need for comprehensive injury surveillance systems for evaluating injury prevention initiatives in sport.

\section{INTRODUCTION}

Rugby union is a type of full contact football most commonly played between two teams of 15 players. Spinal cord injuries, although rare on the basis of exposure per player, are a major cause of serious morbidity and mortality in rugby. ${ }^{1}$ During the 1970 s and 1980s an increase in the reported frequency of catastrophic spinal injuries associated with rugby was documented in medical journals from several countries in which rugby is a popular sport. Since the 1980s and 1990s measures to prevent injury have included changes to laws on scrum procedures, stricter application of existing laws, and educational initiatives. ${ }^{23}$

A review of papers published up to 2001 reported that $40 \%$ of spinal injuries occurring in rugby were the result of the scrum, $36 \%$ were from the tackle, and $18 \%$ from the ruck/maul (see bmj.com for a glossary of terms). The definition of injury used in the studies reviewed, however, varied from admissions to spinal units through to tetraplegia. ${ }^{1}$

Ascertaining the numbers of spinal injuries occurring in rugby and the risks faced by players both in the scrum and in other facets of the game has been hampered by the relative rarity of the events and a lack of standardised procedures for collecting data. ${ }^{134}$ A further impediment to evaluating the risks of spinal injuries in rugby has been a lack of reliable "denominator" data - the number and exposure of participants from which the cases result over a specified period. ${ }^{4}$

A recent call by a consultant general surgeon in the United Kingdom to ban the rugby scrum generated a 